\title{
Pre-transplant portal vein thrombosis in non-alcoholic fatty liver disease patients-pathogenesis, risk factors, and implications on management
}

\author{
Peter DeLeeuw ${ }^{1}$, Uchenna Agbim ${ }^{2}$ \\ ${ }^{1}$ Department of Internal Medicine, University of Tennessee Health Science Center, Memphis, TN, USA; ${ }^{2}$ Department of Surgery, University of \\ Tennessee Health Science Center, Memphis, TN, USA \\ Contributions: (I) Conception and design: U Agbim; (II) Administrative support: None; (III) Provision of study materials or patients: Both authors; (IV) \\ Collection and assembly of data: Both authors; (V) Data analysis and interpretation: Both authors; (VI) Manuscript writing: Both authors; (VII) Final \\ approval of manuscript: Both authors. \\ Correspondence to: Uchenna Agbim, MD. 910 Madison Ave. 2nd Floor, Memphis, TN 38163, USA. Email: uchenna.agbim@gmail.com.
}

\begin{abstract}
Along with the worldwide increase in obesity and metabolic syndrome, non-alcoholic fatty liver disease (NAFLD) and its more severe subset, non-alcoholic steatohepatitis (NASH), are on path to become the leading cause of liver transplantation in the United States. NAFLD, as well as obesity, create an inflammatory milieu via the release of adipocytokines. In turn, the inflammatory environment can trigger an increase in prothrombotic factors. Independent of inflammation, the severity of NASH is associated with a graded increase in hypercoagulability such as an increase in factor VIII, increase in plasminogen activator inhibitor-1, and decrease in protein C. Ultimately, this environment creates an increase in thrombotic risk, leading to higher rates of pre-transplant portal vein thrombosis (PVT) in patients with NASH cirrhosis vesus other causes of cirrhosis. Many studies have shown worse outcomes in liver transplant recipients with PVT as it complicates anastomotic reconstruction which can negatively affect portal blood supply needed for adequate liver functioning. Management and treatment of PVT is not standardized, but from a pharmacologic standpoint, multiple classes of anticoagulants have shown to be successful in recanalization of the portal vein and preventing recurrence of clot with minimal bleeding complications. The increasing prevalence of NASH cirrhosis and subsequent increase in PVT require further research for improved outcomes.
\end{abstract}

Keywords: Metabolic syndrome; non-alcoholic fatty liver disease (NAFLD); portal vein thrombosis (PVT); obesity

Received: 10 December 2019; Accepted: 20 July 2020; Published: 25 July 2022.

doi: $10.21037 /$ tgh-19-361

View this article at: http://dx.doi.org/10.21037/tgh-19-361

\section{Introduction}

In the past decade, the prevalence of non-alcoholic fatty liver disease (NAFLD) has surged worldwide. Nonalcoholic steatohepatitis (NASH), the inflammatory component of NAFLD, now ranks as a leading cause of liver transplantation in the United States. NASH and associated risk factors such as obesity, type 2 diabetes mellitus (T2DM), and hypertension lead to an inflammatory environment, ultimately predisposing patients, particularly those with cirrhosis, to prothrombotic events including portal vein thrombosis (PVT). PVT remains an exceedingly common finding in those with cirrhosis. Emerging evidence from singlecenter and database studies demonstrate NASH patients with cirrhosis and PVT have inferior peri-transplant outcomes. Herein, we discuss the impact of PVT on liver transplantation outcomes in patients with NASH cirrhosis.

\section{Epidemiology of NAFLD/NASH}

Over the last few decades, the prevalence of NAFLD has 
increased worldwide, paralleling the increase in the obesity epidemic. Obesity, T2DM, and the metabolic syndrome are frequent concomitant conditions associated with NAFLD, particularly NASH, the latter which is identified by the histologic presence of steatosis with lobular inflammation and hepatocellular balloon degeneration (1). The global prevalence of NAFLD is estimated to be $25 \%$, with the highest prevalence in South America and the Middle East and the lowest prevalence in Africa (2). NAFLD has varying degrees of severity ranging from simple steatosis to NASH and can lead to progressive fibrosis, ultimately causing cirrhosis and complications of portal hypertension and hepatocellular carcinoma (HCC) (1). It is currently the second leading indication for placement on the liver transplant waiting list (3) and will most likely become the leading cause within the next decade (4).

\section{NAFLD as an inflammatory and prothrombotic disorder}

NAFLD and in particular, NASH, is closely linked to insulin resistance, and as such is commonly seen in patients with obesity and T2DM. It is well established that obesity results in both chronic inflammation and impaired fibrinolysis, ultimately leading to thrombosis (5-8). Abdominal visceral adipose tissue acts as an endocrine organ, releasing hormones, cytokines, and proinflammatory factors that affect the physiology of hemostasis (5). For instance, C-reactive protein, interleukin-6 (IL-6), IL-8, tumor necrosis factor- $\alpha$, and transforming growth factor- $\beta$ are upregulated by adipocytes and macrophages, ultimately stimulating an inflammatory milieu in locations such as the liver and vascular endothelium, all of which contribute to insulin resistance (5-8). Similarly, evidence suggests that NAFLD itself is strongly associated with an inflammatory state independent of obesity (8). It has been proposed that in NAFLD, the liver functions as both a source and target of the adipocytokines, since hepatic steatosis leads to further intrahepatic inflammation exacerbating insulin resistance (9). Additionally, the inflammatory effect of these adipocytokines triggers procoagulant factors, with upregulation of tissue factor and factor VII, ultimately favoring thrombosis (10).

Furthermore, it is widely recognized that obesity and NASH result in increased levels of factor VIII, factor IX, factor XI, factor XII (11), and plasminogen activator inhibitor-1 (PAI-1) $(5,7,12)$. PAI-1 is a critical player in fibrinolysis, as it serves as an inhibitor to tissue plasminogen activator and urokinase, thereby preventing the conversion of plasminogen to plasmin and subsequent breakdown of a fibrin clot $(5,7,12)$. Interestingly, Verrijken et al. (12) noted a graded increase in PAI-1 levels as the histological severity of NASH (determined by the NAFLD activity score) increased, although the authors did not find a correlation with NAFLD and other prothrombotic factor levels. Tripodi et al. (13) demonstrated that the endogenous thrombin potential ratio (a measure of procoagulant imbalance, with higher ratios representing greater procoagulant imbalance), increased in a graded response as the severity of NASH increased. That is, it was lowest in controls, followed by patients with simple steatosis, NASH, and cirrhosis (13). Taken together, these studies indicate a progressive increase in prothrombotic factors that parallels the severity of NAFLD, with those without NAFLD having low levels, followed by intermediate levels in those with simple steatosis and the highest levels in those with biopsyproven NASH and cirrhosis $(7,9,12,13)$.

\section{Epidemiology of PVT and its complications in cirrhosis}

Emerging evidence reveals a reduction in both antithrombotic and prothrombotic factors in patients with cirrhosis creating a new, albeit unstable "rebalance", with manifestations of this rebalance of hemorrhage or thrombosis molded by situational conditions (spontaneous bacterial peritonitis, hepatorenal syndrome etc.). Despite this "rebalance", mounting evidence shows patients with cirrhosis produce a greater amount of thrombin than patients without cirrhosis, owing to a concomitant increase in factor VIII and decrease in protein C; overall this favors a pro-coagulant state $(14,15)$. In fact, patients with cirrhosis and PVT have been noted to have decreased protein C, protein $\mathrm{S}$, and antithrombin compared to patients with cirrhosis and no evidence of PVT (16).

These systemic factors favoring hypercoagulability, along with increased intrahepatic resistance from fibrosis and endothelial injury, play a role in formation of thrombosis $(16,17)$. Perhaps most importantly, hemodynamic factors such as decreased portal flow velocity contribute to PVT development (16). Other risk factors associated with PVT development in patients with cirrhosis include previous abdominal surgery, procedures such as balloon-occluded retrograded transvenous obliteration or those employed to treat HCC such as percutaneous liver directed tumor therapy, and HCC itself, which can cause vascular invasion 
into the portal vein, as well as contribute to non-neoplastic PVT $(17,18)$. Ascites, encephalopathy, low platelets, low hemoglobin, decompensated liver disease, increased spleen thickness, portal vein diameter, and diabetes are also associated with PVT $(16,19,20)$. Thus, it is no wonder that PVT is a common complication in cirrhosis with a prevalence of $2-26 \%$ in candidates awaiting liver transplantation (17). According to a meta-analysis, adults with cirrhosis and PVT have a 2 -fold increased risk of developing ascites and a $62 \%$ increased risk of mortality compared to patients with cirrhosis without PVT (21).

PVT may be incidentally detected via cross-sectional imaging routinely performed for HCC surveillance or noted upon imaging ordered during decompensating episodes (19). Abdominal ultrasound with color doppler has a sensitivity and specificity of $80-100 \%$. Contrast enhanced ultrasound can increase both sensitivity and specificity to $88-100 \%$ and $94-96 \%$, respectively (22). Computed tomography and magnetic resonance imaging both demonstrate superiority when compared to ultrasound, as the former modes do not rely on operator-dependency, can evaluate the extent of thrombosis, and can provide additional features regarding liver parenchyma (22).

Ample evidence indicates PVT affects peri-transplant morbidity and mortality. One retrospective study using the Organ Procurement and Transplant Network (OPTN) database found the prevalence of PVT at candidate registration increased between 2002 and 2014, and the presence of PVT was associated with increased waitlist dropout due to illness severity (23). Ghabril et al. (24) reported that interval development of PVT between waitlist registration and liver transplantation was associated with longer wait times and a higher model for end-stage liver disease (MELD) score increase. Of those who had PVT, only $31 \%$ of the patients were diagnosed at listing (24). Others report up to $50 \%$ of transplant recipients may have unrecognized PVT until the time of transplantation (19). These findings highlight that interval development of PVT is common and indicate that new PVT at liver transplantation is associated with progression of underlying liver disease. Furthermore, in the same aforementioned Ghabril et al. study, PVT was associated with an increased 90-day mortality and graft failure post-transplantation (24). Similarly, a 2018 meta-analysis of 44 studies noted the 30-day and 1-year post-transplant mortality in those with PVT was significantly higher than those without PVT (25).

Characterizing the degree of occlusion and extent of propagation is important for management and prognosis, particularly since occlusive PVTs result in high peritransplant complications (26-28). The Yerdel classification characterizes lumen occlusion and extent of thrombosis into four grades (29) with higher grades [3-4] often requiring complex vascular reconstructions (19). In a small study of 25 patients with PVT and no evidence of HCC who underwent transplant, PVT grade correlated with poor patient survival regardless of surgical technique (30). A larger, single-center study of over 3,200 liver transplant candidates noted those undergoing transplant with occlusive PVT had a sevenfold increased risk of 30-day mortality in comparison to those without PVT (26). Another retrospective study from a single institution showed that total occlusion of PVT had a higher mortality, whereas patients with partial occlusion had similar survival rates as patients without PVT (27). In a study of liver transplant recipients from Canada and the United Kingdom, Karvellas et al. also noted patients with complete PVT at liver transplantation and those with reocclusion in the post-transplant setting had suboptimal outcomes (28).

\section{PVT in patients with NASH cirrhosis}

While the exact mechanisms underlying an increase of PVT in patients with NASH have not been elucidated, the previously mentioned body of evidence underscoring the role of inflammatory and prothrombotic biomarkers cannot be ignored (Figure 1). Epidemiologic data show patients with NASH cirrhosis and obesity have an amplified risk of PVT. In a review of the OPTN database, Stine et al. (31) document that there was an increased prevalence of PVT at liver transplantation among patients with NASH compared to all other indications $(10.1 \%$ vs. $6.0 \% ; \mathrm{P}<0.001)$. Moreover, NASH cirrhosis was the strongest risk factor associated with a diagnosis of PVT at liver transplantation (31). In a separate study, these same authors noted liver transplant recipients with $\mathrm{NASH}$ whom had high risk demographic and clinical features [age $>60$, body mass index $(\mathrm{BMI})>30 \mathrm{~kg} / \mathrm{m}^{2}$, and history of pre-transplant T2DM or hypertension] were twice as likely to have PVT at liver transplantation (32). Similarly, other database and singlecenter studies have noted NASH, obesity, BMI, and T2DM as risk factors for PVT development (23,33), and it has been noted that obesity, T2DM, and cryptogenic cirrhosis (the latter diagnosis is commonly considered as undiagnosed NAFLD) were risk factors for interval development of PVT between transplant listing and transplantation (24). In addition to an increased risk of PVT among liver transplant 


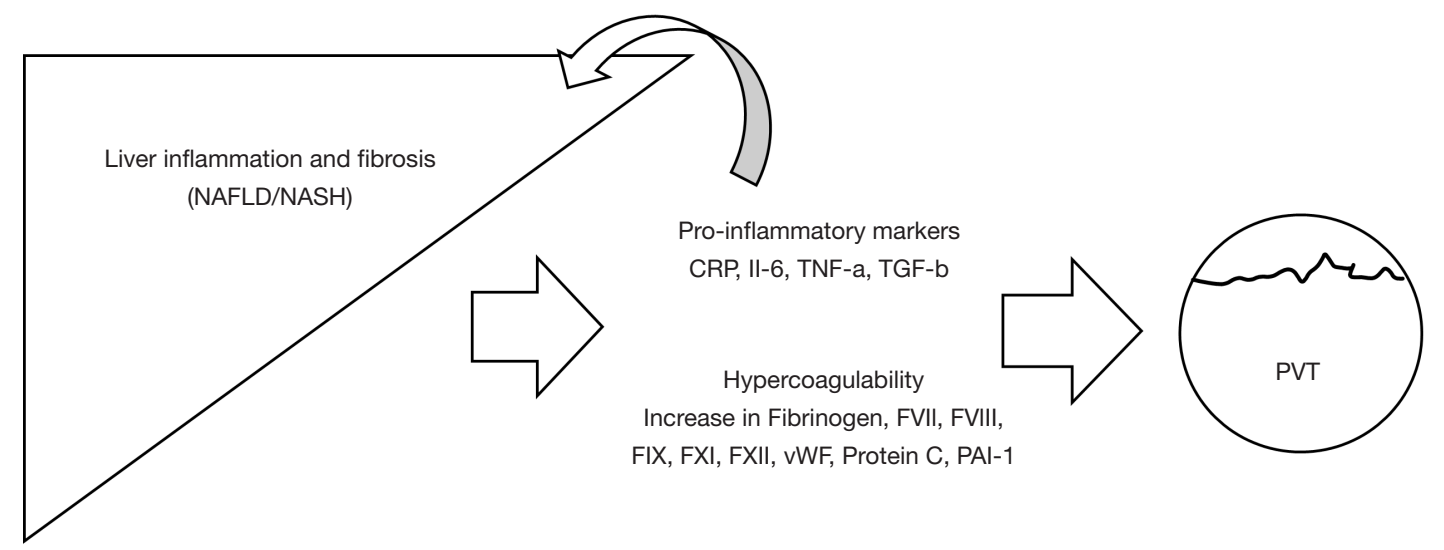

Figure 1 NAFLD/NASH leading to PVT. The independent pathogenesis of NAFLD/NASH creates a proinflammatory and prothrombotic state, which not only creates an environment favoring thrombosis including PVT. Adapted from Targher et al. (7). NAFLD, non-alcoholic fatty liver disease; NASH, non-alcoholic steatohepatitis; CRP, C-reactive protein; vWF, von Willebrand factor; PVT, portal vein thrombosis.

Table 1 Benefits and drawbacks of PVT treatment options

\begin{tabular}{lll}
\hline PVT therapeutic options & Specific benefits & Drawbacks \\
\hline LMWH & $\begin{array}{l}\text { No lab monitoring, fixed dose, does not } \\
\text { affect MELD score, reversible }\end{array}$ & $\begin{array}{l}\text { Needs to be renally adjusted, no oral administration, concern for } \\
\text { effectiveness in advanced cirrhosis }\end{array}$ \\
VKA & Low cost, oral administration, reversible & $\begin{array}{l}\text { Monitoring of INR required and affects MELD score } \\
\text { DOAC }\end{array}$ \\
NIPS & $\begin{array}{l}\text { affects MELD score, oral administration } \\
\text { No anticoagulation }\end{array}$ & $\begin{array}{l}\text { Reversible agents not always available, hepatotoxicity in } \\
\text { rivaroxaban }\end{array}$ \\
& & $\begin{array}{l}\text { Procedure experience required, failure or shunt dysfunction, } \\
\text { hepatic encephalopathy, liver failure, can complicate transplant } \\
\text { surgery }\end{array}$ \\
\hline
\end{tabular}

Table adapted from Loudin et al. (36). PVT, portal vein thrombosis; LMWH, low molecular weight heparin; VKA, vitamin K antagonist; DOAC, direct oral anticoagulant; TIPS, transjugular intrahepatic portosystemic shunt; MELD, model for end-stage liver disease; INR, international normalized ratio.

registrants with NASH, liver transplant recipients with NASH cirrhosis and pre-transplant PVT had a $45 \%$ increased risk of 90-day graft failure compared to patients with NASH and no evidence of PVT at transplant (34).

\section{Management of PVT}

As the frequency of NASH cirrhosis increases, it is likely that clinicians will see more cases of PVT in this population $(31,35)$. Similarly, since NAFLD is associated with an increase in arterial and venous thrombosis, comprehensive approaches such as treatment with antithrombotic agents will be required (35). At present, no strategies specific to liver transplant candidates with NASH and PVT exist. However, since patients with NASH cirrhosis that develop
PVT represent a high-risk subgroup in terms of posttransplant morbidity, particular attention should be taken to optimize these patients' outcomes. Restoration of portal flow results in improvement in many of the previously mentioned risks including peri-transplant surgical complications and recanalization prevents extension into the mesenteric/splenic venous system (35). Table 1 summarizes various treatment strategies.

\section{Pharmacotherapy}

In general, anticoagulation helps prevent propagation of clot and is thought to halt intrahepatic microthrombi leading to ischemia and subsequent cell death, a term known as parenchymal extinction $(10,37)$. Studies have examined 
anticoagulation prior to transplantation with low molecular weight heparin (LMWH) and vitamin $\mathrm{K}$ antagonists (VKAs) as both prevention and/or treatment of PVT. One Spanish study of 55 patients with cirrhosis found that both LMWH and VKAs produced partial or complete recanalization in $60 \%$ of patients with early initiation significantly affecting recanalization. Re-thrombosis was seen in $38.5 \%$ once anticoagulation had been stopped, which lead the authors to recommend continuing anticoagulation indefinitely. Although 5 patients on anticoagulation did have bleeding complications, a platelet count of less than $50 \times 10^{9} / \mathrm{L}$ was the only significant factor associated with having a higher risk of bleeding complications (38). In one small unblinded, randomized control trial out of Italy, a 12-month course of LMWH prevented PVT in patients with cirrhosis and a Child-Pugh score of 7-10. No adverse side effects or hemorrhagic events were identified. It also appeared that the patients taking $\mathrm{LMWH}$ had a delay in hepatic decompensation and improved survival (37). Another small Italian study of 28 patients looking at the effectiveness of enoxaparin in the resolution of PVT in patients with cirrhosis found a complete response in $75 \%$ of patients. Of note, there were no adverse effects such as bleeding (39). A more recent meta-analysis of patients with cirrhosis and PVT evaluated anticoagulation with LMWH or warfarin versus no anticoagulation therapy. The results showed more recanalization $(71 \%$ vs. $42 \% ; \mathrm{P}<0.0001)$ and less progression of PVT (odds ratio: 0.141 ; $95 \%$ confidence interval, $0.06-0.31 ; \mathrm{P}<0.001)$ in patients on anticoagulation compared to those not treated with anticoagulation. There was also no increase in major or minor bleeding in the anticoagulated patients; in fact, these patients had a decrease in variceal hemorrhage (40). With the exception of the meta-analysis, the studies to date have been small in nature, but taken together, the results show that both $\mathrm{LMWH}$ and VKAs are safe to use and beneficial in patients with cirrhosis and PVT. Nevertheless, larger controlled trials are needed for anticoagulation to become a mainstay for prevention and individualized patient care should be taken when considering anticoagulation for treatment of PVT.

\section{$L M W H$}

While the optimal anticoagulant agent to use for PVT has not been firmly established, LMWH has been shown to be safe and effective in patients with cirrhosis. LMWH indirectly inhibits factor $\mathrm{Xa}$. Unlike VKAs, there is no required lab monitoring with $\mathrm{LMWH}$, although some evidence suggest anti-Xa levels can be used as a proxy for LMWH concentration (41), but data are conflicting $(35,42)$. Furthermore, it does not affect the MELD score and can be administered in a fixed twice daily subcutaneous dose adjusted for body weight to treat PVT. LMWH does have some drawbacks. The medication dose requires adjustment depending on renal function, is not available in oral form, is only partially reversed with protamine, can cause heparininduced thrombocytopenia, and is more expensive than older medications like VKAs. There is also concern in the effectiveness of $\mathrm{LMWH}$ in advanced cirrhosis due to impaired synthesis of antithrombin $(19,35,41,42)$.

\section{$V K A s$}

VKAs such as warfarin inhibit a vitamin $\mathrm{K}$ dependent enzyme responsible for synthesis of factors II, VII, IX, and $\mathrm{X}$. VKAs are beneficial due to their low cost, ease of administration given it is an oral medication, and reversibility. Unfortunately, VKAs require international normalized ratio (INR) monitoring to maintain therapeutic levels. Not only does this create issues with monitoring compliance, but it is difficult to determine therapeutic INR levels in patients with cirrhosis as the INR is already elevated. INR is also a component of the MELD score, meaning that initiation of VKAs can raise the MELD score, subsequently affecting priority for liver transplant $(19,35,42)$.

\section{Direct oral anticoagulants (DOACs)}

DOACs which either directly inhibit factor Xa (rivaroxaban, apixaban, edoxaban, and betrixaban) or directly inhibit thrombin (dabigatran) have not been rigorously studied in patients with cirrhosis. In fact, patients with cirrhosis were often excluded from the initial drug trials. While these medications have increasing utilization in a variety of conditions and have the benefit of fixed dosing and oral administration, they are expensive and the reversal agents are not universally available as are the antidotes for VKA or LMWH (43). There have been limited studies showing partial or complete recanalization of the portal vein with the use of DOACs. A recent study comparing edoxaban versus warfarin for 6 months in patients with cirrhosis and acute PVT showed the edoxaban group had a higher percentage of complete resolution of PVT (70\% vs. $20 \%$; $\mathrm{P}<0.001)$. In addition, a smaller percentage of patients on edoxaban had progression of PVT ( $5 \%$ vs. $47 \% ; \mathrm{P}<0.01)$, defined 
as increase in the PVT volume post-treatment compared to the pre-treatment PVT volume. While this study is interesting, it did not have enough power to evaluate for a difference in bleeding complications $(43,44)$. The European Association for the Study of the Liver guidelines recommend caution when using DOACs due to lack of research, as well as cases of hepatotoxicity with the use of rivaroxaban (42).

\section{Invasive interventions}

\section{Transjugular intrabepatic portal shunt (TIPS)}

PVT was once seen as a contraindication for TIPS. Now, TIPS is often performed for those with PVT and complications of portal hypertension (variceal hemorrhage, ascites) and can be considered a treatment option for PVT, as return of portal blood flow via re-canalization of the portal vein can prevent further thrombosis. The success of TIPS is often limited by the extent of the thrombus and experience of the performing center $(19,45,46)$. Senzolo et al. demonstrated that TIPS can result in recanalization and decrease thrombosis extension in patients who had contraindications to anticoagulation or who had progression of clot in the setting of anticoagulation (47). When post-TIPS anticoagulation therapy has been studied, there appears to be comparable recanalization rates when comparing patients without anticoagulation, suggesting that anticoagulation is not needed for continued portal vein patency $(19,46)$. The disadvantages of TIPS include operator inexperience, shunt dysfunction or need for revision, hepatic encephalopathy, and hepatic failure (19).

\section{Thrombolysis}

Thrombolysis has been looked at as a treatment option for acute PVT, however studies have shown that morbidity and complication rates are high. As such, the procedure is not usually recommended for chronic PVT. An alternative to thrombolysis is mechanical thrombectomy. Although mechanical thrombectomy is a technique that rapidly removes the clot and avoids thrombolytic medications, it can cause damage to the intimal or vascular layers of the portal vein, increasing the risk of recurrent thrombosis (48).

\section{Key points}

(I) NAFLD/NASH is an increasing cause of liver disease that has an increased hypercoagulable state, higher than other liver diseases;

(II) This increased prothrombotic risk leads to higher rates of PVT;

(III) More research is needed to standardize treatment of PVT with anticoagulation or TIPS procedure;

(IV) PVT without treatment leads to transplant surgery complications and worse outcomes post-transplant.

\section{Conclusions}

In conclusion, NAFLD/NASH is an increasing cause of liver disease that has a higher risk of hypercoagulability and therefore higher rates of PVT than other etiologies of cirrhosis. PVT has been shown to complicate the liver transplant operation and worsen outcomes after liver transplant. Therefore, treatment will become important as the prevalence of both NASH cirrhosis and PVT increase. Anticoagulation and TIPS procedure can benefit patients and, in some cases, resolve PVT, but more research is required to make strong conclusions about their widespread use in patients with PVT and cirrhosis due to NAFLD.

\section{Acknowledgments}

Funding: None.

\section{Footnote}

Provenance and Peer Review: This article was commissioned by the Guest Editors (Sanjaya K. Satapathy, David Bernstein, Nitzan Roth) for the series "Liver Transplantation in NASH and ALD" published in Translational Gastroenterology and Hepatology. The article has undergone external peer review.

Conflicts of Interest: Both authors have completed the ICMJE uniform disclosure form (available at https://tgh. amegroups.com/article/view/10.21037/tgh-19-361/coif). The series "Liver Transplantation in NASH and ALD" was commissioned by the editorial office without any funding or sponsorship. The authors have no other conflicts of interest to declare.

Ethical Statement: The authors are accountable for all aspects of the work in ensuring that questions related to the accuracy or integrity of any part of the work are 
appropriately investigated and resolved.

Open Access Statement: This is an Open Access article distributed in accordance with the Creative Commons Attribution-NonCommercial-NoDerivs 4.0 International License (CC BY-NC-ND 4.0), which permits the noncommercial replication and distribution of the article with the strict proviso that no changes or edits are made and the original work is properly cited (including links to both the formal publication through the relevant DOI and the license). See: https://creativecommons.org/licenses/by-nc-nd/4.0/.

\section{References}

1. Chalasani N, Younossi Z, Lavine JE, et al. The diagnosis and management of nonalcoholic fatty liver disease: Practice guidance from the American Association for the Study of Liver Diseases. Hepatology 2018;67:328-57.

2. Younossi ZM, Koenig AB, Abdelatif D, et al. Global epidemiology of nonalcoholic fatty liver disease-Metaanalytic assessment of prevalence, incidence, and outcomes. Hepatology 2016;64:73-84.

3. Wong RJ, Aguilar M, Cheung R, et al. Nonalcoholic steatohepatitis is the second leading etiology of liver disease among adults awaiting liver transplantation in the United States. Gastroenterology 2015;148:547-55.

4. Charlton MR, Burns JM, Pedersen RA, et al. Frequency and outcomes of liver transplantation for nonalcoholic steatohepatitis in the United States. Gastroenterology 2011;141:1249-53.

5. Targher G, Chonchol M, Miele L, et al. Nonalcoholic fatty liver disease as a contributor to hypercoagulation and thrombophilia in the metabolic syndrome. Semin Thromb Hemost 2009;35:277-87.

6. Alessi MC, Juhan-Vague I. Metabolic syndrome, haemostasis and thrombosis. Thromb Haemost 2008;99:995-1000.

7. Targher G, Byrne CD. Diagnosis and management of nonalcoholic fatty liver disease and its hemostatic/ thrombotic and vascular complications. Semin Thromb Hemost 2013;39:214-28.

8. Targher G, Zoppini G, Moghetti P, et al. Disorders of coagulation and hemostasis in abdominal obesity: emerging role of fatty liver. Semin Thromb Hemost 2010;36:41-8.

9. Anstee QM, Targher G, Day CP. Progression of NAFLD to diabetes mellitus, cardiovascular disease or cirrhosis. Nat Rev Gastroenterol Hepatol 2013;10:330-44.

10. Anstee QM, Wright M, Goldin R, et al. Parenchymal extinction: coagulation and hepatic fibrogenesis. Clin Liver Dis 2009;13:117-26.

11. Kotronen A, Joutsi-Korhonen L, Sevastianova K, et al. Increased coagulation factor VIII, IX, XI and XII activities in non-alcoholic fatty liver disease. Liver Int 2011;31:176-83.

12. Verrijken A, Francque S, Mertens I, et al. Prothrombotic factors in histologically proven nonalcoholic fatty liver disease and nonalcoholic steatohepatitis. Hepatology 2014;59:121-9.

13. Tripodi A, Fracanzani AL, Primignani M, et al. Procoagulant imbalance in patients with non-alcoholic fatty liver disease. J Hepatol 2014;61:148-54.

14. Tripodi A, Mannucci PM. The coagulopathy of chronic liver disease. N Engl J Med 2011;365:147-56.

15. Tripodi A, Anstee QM, Sogaard KK, et al. Hypercoagulability in cirrhosis: causes and consequences. J Thromb Haemost 2011;9:1713-23.

16. Zocco MA, Di Stasio E, De Cristofaro R, et al. Thrombotic risk factors in patients with liver cirrhosis: correlation with MELD scoring system and portal vein thrombosis development. J Hepatol 2009;51:682-9.

17. Ponziani FR, Zocco MA, Senzolo M, et al. Portal vein thrombosis and liver transplantation: implications for waiting list period, surgical approach, early and late followup. Transplant Rev (Orlando) 2014;28:92-101.

18. Ravaioli $M$, Zanello $M$, Grazi GL, et al. Portal vein thrombosis and liver transplantation: evolution during 10 years of experience at the University of Bologna. Ann Surg 2011;253:378-84.

19. Francoz C, Valla D, Durand F. Portal vein thrombosis, cirrhosis, and liver transplantation. J Hepatol 2012;57:203-12.

20. Abdel-Razik A, Mousa N, Elhelaly R, et al. De-novo portal vein thrombosis in liver cirrhosis: risk factors and correlation with the Model for End-stage Liver Disease scoring system. Eur J Gastroenterol Hepatol 2015;27:585-92.

21. Stine JG, Shah PM, Cornella SL, et al. Portal vein thrombosis, mortality and hepatic decompensation in patients with cirrhosis: A meta-analysis. World J Hepatol 2015;7:2774-80.

22. Alzubaidi S, Patel I, Saini A, et al. Current concepts in portal vein thrombosis: etiology, clinical presentation and management. Abdom Radiol (NY) 2019;44:3453-62.

23. Montenovo M, Rahnemai-Azar A, Reyes J, et al. Clinical Impact and Risk Factors of Portal Vein Thrombosis for Patients on Wait List for Liver Transplant. Exp Clin Transplant 2018;16:166-71.

24. Ghabril M, Agarwal S, Lacerda M, et al. Portal Vein 
Thrombosis Is a Risk Factor for Poor Early Outcomes After Liver Transplantation: Analysis of Risk Factors and Outcomes for Portal Vein Thrombosis in Waitlisted Patients. Transplantation 2016;100:126-33.

25. Zanetto A, Rodriguez-Kastro KI, Germani G, et al. Mortality in liver transplant recipients with portal vein thrombosis - an updated meta-analysis. Transpl Int 2018;31:1318-29.

26. Englesbe MJ, Kubus J, Muhammad W, et al. Portal vein thrombosis and survival in patients with cirrhosis. Liver Transpl 2010;16:83-90.

27. Suarez Artacho G, Barrera Pulido L, Alamo Martinez JM, et al. Outcomes of liver transplantation in candidates with portal vein thrombosis. Transplant Proc 2010;42:3156-8.

28. Karvellas CJ, Cardoso FS, Senzolo M, et al. Clinical Impact of Portal Vein Thrombosis Prior to Liver Transplantation: A Retrospective Cohort Study. Ann Hepatol 2017;16:236-436.

29. Yerdel MA, Gunson B, Mirza D, et al. Portal vein thrombosis in adults undergoing liver transplantation: risk factors, screening, management, and outcome. Transplantation 2000;69:1873-81.

30. Fouzas I, Paul A, Becker C, et al. Orthotopic liver transplantation in patients with portal vein thrombosis in the absence of hepatocellular carcinoma. Transplant Proc 2012;44:2734-6.

31. Stine JG, Shah NL, Argo CK, et al. Increased risk of portal vein thrombosis in patients with cirrhosis due to nonalcoholic steatohepatitis. Liver Transpl 2015;21:1016-21.

32. Stine JG, Argo CK, Pelletier SJ, et al. Advanced nonalcoholic steatohepatitis cirrhosis: A high-risk population for pre-liver transplant portal vein thrombosis. World J Hepatol 2017;9:139-46.

33. Ayala R, Grande S, Bustelos R, et al. Obesity is an independent risk factor for pre-transplant portal vein thrombosis in liver recipients. BMC Gastroenterol 2012;12:114.

34. Agbim U, Jiang Y, Kedia SK, et al. Impact of Nonmalignant Portal Vein Thrombosis in Transplant Recipients With Nonalcoholic Steatohepatitis. Liver Transpl 2019;25:68-78.

35. Lisman T, Kamphuisen PW, Northup PG, et al. Established and new-generation antithrombotic drugs in patients with cirrhosis - possibilities and caveats. J Hepatol 2013;59:358-66.

36. Loudin M, Ahn J. Portal Vein Thrombosis in Cirrhosis. J Clin Gastroenterol 2017;51:579-85.

37. Villa E, Camma C, Marietta M, et al. Enoxaparin prevents portal vein thrombosis and liver decompensation in patients with advanced cirrhosis. Gastroenterology 2012;143:1253-60.e4.

38. Delgado MG, Seijo S, Yepes I, et al. Efficacy and safety of anticoagulation on patients with cirrhosis and portal vein thrombosis. Clin Gastroenterol Hepatol 2012;10:776-83.

39. Amitrano L, Guardascione MA, Menchise A, et al. Safety and efficacy of anticoagulation therapy with low molecular weight heparin for portal vein thrombosis in patients with liver cirrhosis. J Clin Gastroenterol 2010;44:448-51.

40. Loffredo L, Pastori D, Farcomeni A, et al. Effects of Anticoagulants in Patients With Cirrhosis and Portal Vein Thrombosis: A Systematic Review and Meta-analysis. Gastroenterology 2017;153:480-7.e1.

41. Bechmann LP, Sichau M, Wichert M, et al. Lowmolecular-weight heparin in patients with advanced cirrhosis. Liver Int 2011;31:75-82.

42. European Association for the Study of the Liver. EASL Clinical Practice Guidelines: Vascular diseases of the liver. J Hepatol 2016;64:179-202.

43. Weinberg EM, Palecki J, Reddy KR. Direct-Acting Oral Anticoagulants (DOACs) in Cirrhosis and CirrhosisAssociated Portal Vein Thrombosis. Semin Liver Dis 2019;39:195-208.

44. Nagaoki Y, Aikata H, Daijyo K, et al. Efficacy and safety of edoxaban for treatment of portal vein thrombosis following danaparoid sodium in patients with liver cirrhosis. Hepatol Res 2018;48:51-8.

45. Salem R, Vouche M, Baker T, et al. Pretransplant Portal Vein Recanalization-Transjugular Intrahepatic Portosystemic Shunt in Patients With Complete Obliterative Portal Vein Thrombosis. Transplantation 2015;99:2347-55.

46. Wang Z, Jiang MS, Zhang HL, et al. Is Post-TIPS Anticoagulation Therapy Necessary in Patients with Cirrhosis and Portal Vein Thrombosis? A Randomized Controlled Trial. Radiology 2016;279:943-51.

47. Senzolo M, M Sartori T, Rossetto V, et al. Prospective evaluation of anticoagulation and transjugular intrahepatic portosystemic shunt for the management of portal vein thrombosis in cirrhosis. Liver Int 2012;32:919-27.

48. Uflacker R. Applications of percutaneous mechanical thrombectomy in transjugular intrahepatic portosystemic shunt and portal vein thrombosis. Tech Vasc Interv Radiol 2003;6:59-69.

doi: $10.21037 /$ tgh-19-361

Cite this article as: DeLeeuw P, Agbim U. Pre-transplant portal vein thrombosis in non-alcoholic fatty liver disease patientspathogenesis, risk factors, and implications on management. Transl Gastroenterol Hepatol 2022;7:27. 